\title{
Design of Class-E ZVS Inverter With Loosely-Coupled Transformer at Fixed Coupling Coefficient
}

\author{
Fabio Corti, Francesco Grasso, and \\ Alberto Reatti \\ DINFO-University of Florence \\ Via Santa Marta 3 I-50139 Florence \\ \{fabio.corti\}@stud.unifi.it \\ \{francesco.grasso, alberto.reatti\}@unifi.it
}

\author{
Agasthya Ayachit, Dalvir K. Saini, and \\ Marian K. Kazimierczuk \\ Department of Electrical Engineering \\ Wright State University \\ 3640 Colonel Glenn Hwy., Dayton, OH, 45435, USA \\ \{ayachit.2, saini.11, marian.kazimierczuk\}@wright.edu
}

\begin{abstract}
The design of Class-E zero-voltage switching (ZVS) inverter with a loosely-coupled transformer is introduced in this paper. In the presented approach, the magnetizing and leakage inductances of the transformer are absorbed into the main circuit. The synthesis of the transformer-version of Class- $\mathrm{E} \mathrm{ZVS}$ inverter into its equivalent $\pi 2 a$ topology is presented. The $\pi 2 a$ topology improves the range of the optimum load resistance required to achieve ZVS. An example of the Class-E inverter with dc supply voltage $10 \mathrm{~V}$, output power $10 \mathrm{~W}$, switching frequency $100 \mathrm{kHz}$ and at a coupling coefficient of 0.77 is considered. The inverter is designed to achieve both zero-voltage switching (ZVS) and zero derivative switching (ZDS) conditions. The analytical expressions are validated through simulation results for an optimum coupling coefficient of 0.77 . In view of potential misalignment between the primary and secondary coils, simulation results are provided for coupling coefficients, which are lower and higher than the optimum value. It is shown that both ZVS and ZDS can be achieved at a coupling coefficient lower than the optimum value and is not possible to achieve ZVS at a coupling coefficient higher than the optimum value. Overall efficiency of $94.3 \%$ is achieved at a coupling coefficient of $0.77,93.4 \%$ at 0.85 , and $92.12 \%$ at 0.7. The presented approach can be used for transformers with reactive load impedances also.
\end{abstract}

\section{INTRODUCTION}

Class-E soft switching power inverters belong to a class of high efficiency electronic circuits [1] - [20]. At an optimum operating point, the Class-E inverters are capable of achieving both zero-voltage switching (ZVS) and zero-derivative switching (ZDS) [1]- [7]. Today's advancements, especially in the area of wireless energy transfer are focused towards improving the transfer efficiency, while being able to achieve a long range for power transmission [5] - [9]. Class-E ZVS inverters have been identified as an ideal solution for these applications.

A Class-E ZVS inverter using a transformer with loosely coupled primary and secondary windings is discussed in this paper. The transformer model considered in this paper includes the magnetizing inductance as well as the primary and secondary-side leakage inductances. The input impedance of a loosely-coupled transformer is a function of coupling coefficient [10] and is represented as a series combination of coupling-dependent resistance and inductance. Thus, the optimum load resistance can be determined for the Class-E ZVS inverter at a fixed coupling coefficient.

The transformer version of the inverter is as shown in Fig. 1(a). The resulting equivalent circuit is identical to the impedance inverter discussed in [1] - [2] and has several benefits over the resistively loaded Class-E ZVS inverter. The impedance inverter is also termed as Class-E inverter with $\pi 2 a$ impedance matching topology. The Class-E ZVS impedance inverter extends the range of load resistance required for optimum operation [6], [7]. This paper introduces the technique required to design the Class-E inverter for optimum operation at a specific coupling coefficient.

\section{Circuit AnAlysis}

\section{A. Circuit Description}

Fig. 1(a) shows the transformer version of the Class-E ZVS resonant inverter. In the circuit, $V_{I}$ is the dc supply voltage, $L_{f}$ is the choke inductor, $S$ is the power MOSFET, and $C_{1 e x t}$ is the voltage-shaping shunt capacitor across the MOSFET including the power MOSFET parasitic ouput capacitance. The transformer is composed of primary self-inductance $L_{p}$, secondary self-inductance $L_{s}$, magnetizing inductance $L_{m}$, and primary and secondary leakage inductances $L_{l p}$ and $L_{l s}$, respectively. The series resonant tank is composed of the resonant capacitor $C$ and the resonant inductor $L_{1}=L_{e x t}+L_{l p}$. A capacitor $C_{s}$ is placed in series with the secondary winding to cancel the reactance of the secondary leakage inductance $L_{l s}$. The turns ratio of the transformer is $n$ and the coupling coefficient is $k$. The load resistance $R_{\text {ir }}$ can be considered as the terminal resistance of the inverter or the equivalent input resistance of a rectifier, required to obtain a dc output voltage. The self capacitance of the inductor and transformer windings are absorbed into the shunt capacitance across the MOSFET.

Assuming that the effect of the $L_{l s}$ is nullified by $C_{s}$, the equivalent impedance of the secondary is equal to $R_{i r}$. Fig. 1(b) shows an equivalent circuit obtained by transferring the load resistance $R_{i r}$ to the primary side to yield $R_{i}$. This circuit is identical to the Class-E ZVS resonant inverter with $\pi 2 a$ 


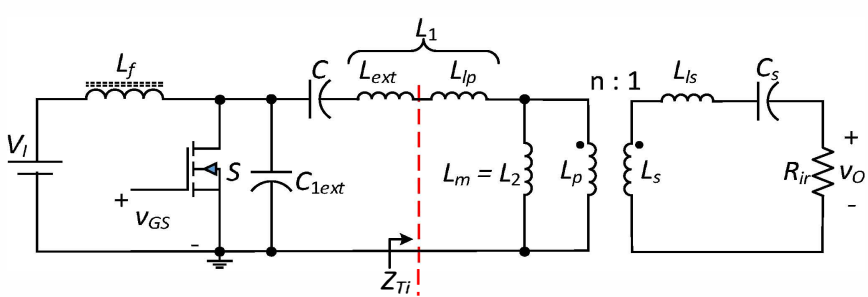

(a)

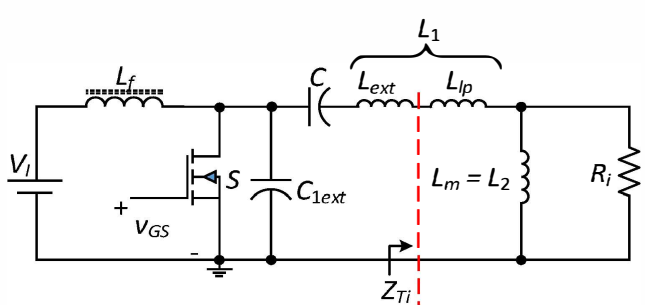

(b)



(c)

Fig. 1. Class-E ZVS inverter. (a) Transformer version. (b) Class-E inverter with $\pi 2 a$ impedance matching circuit. (c) Class-E loaded by series resonant circuit with coupling-dependent load resistor $R_{T i}$ and resonant inductor $L$.

impedance matching network and is discussed in depth in [7]. Since the load to the Class-E circuit is of impedance type, the circuit shown in Fig. 1(a) is commonly referred to as the Class-E impedance inverter.

Since the transformer voltage and current gain are dependent on the coupling coefficient $k$, the input impedance $Z_{T i}$ also varies with $k$. Using the method to determine the input impedance of a transformer discussed in [10], the input impedance can be considered as a series combination of its equivalent inductance $L_{T i}$ and its equivalent resistance $R_{T i}$ and is shown in Fig. 1(c). The circuit shown in Fig. 1 (c) is identical to the classical Class-E ZVS inverter comprising of the resonant capacitor $C$, the resonant inductor $L=L_{e x t}+L_{T i}$, and the load resistance $R_{T i}$.

\section{B. Circuit Design}

The rated output power of the Class-E inverter with transformer shown in Fig. 1(a) is $P_{R i}$. Therefore, the load resistance of the inverter assuming efficiency $\eta=1$ is

$$
R_{i r}=\frac{8}{\pi^{2}+4} \frac{V_{I}^{2}}{P_{R i}} .
$$

Assuming that the self-inductances $L_{p}$ and $L_{s}$ of the primary and secondary windings, respectively are known a priori, the magnetizing inductance is

$$
L_{m}=k L_{p}
$$

The leakage inductances are

$$
\begin{gathered}
L_{l p}=(1-k) L_{p}=\frac{(1-k)}{k} L_{m}, \\
L_{l s}=(1-k) L_{s}=(1-k) \frac{L_{p}}{n^{2}}=\frac{(1-k)}{n^{2} k} L_{m} .
\end{gathered}
$$

The reactances of the secondary-side leakage inductance $L_{l s}$ and series capacitance $C_{s}$ are equal and yields

$$
C_{s}=\frac{1}{\omega^{2} L_{l s}}=\frac{1}{4 \pi^{2} f_{s}^{2} L_{l s}}=\frac{n^{2}}{4 \pi^{2} f_{s}^{2}(1-k) L_{p}},
$$

where $\omega=2 \pi f_{s}$ and $f_{s}$ is the switching frequency of the Class-E inverter. The total resistance of the secondary side is equal to $R_{i r}$. Applying the reflection principle, the resistance $R_{i r}$ can be transferred to the primary side. Thus, the equivalent load resistance referred to the primary side as shown in Fig. $1(b)$ is

$$
R_{i}=n^{2} R_{i r}
$$

The input impedance $Z_{T i}$ is described as [10]

$$
Z_{T i}=s L_{l p}+\left(s L_{m} \| R_{i}\right) .
$$

Substituting (2) and (3) into (7) results in

$$
Z_{T i}=s L_{l p}+\frac{s L_{m} R_{i}}{s L_{m}+R_{i}}=s(1-k) L_{p}+\frac{s k L_{p} R_{i}}{s k L_{p}+R_{i}}
$$

to yield

$$
Z_{T i}=\frac{s^{2}(1-k) k L_{p}^{2}+s L_{p} R_{i}}{s k L_{p}+R_{i}} .
$$

Substituting $s=j \omega$ into (9) yields

$$
\begin{aligned}
Z_{T i} & =R_{T i}+j X_{T i} \\
& =\frac{\omega^{2} L_{p}^{2} k^{2} R_{i}}{R_{i}^{2}+\omega^{2} k^{2} L_{p}^{2}}+j \frac{\omega^{3} L_{p}^{3} k^{2}(1-k)+\omega L_{p} R_{i}^{2}}{R_{i}^{2}+\omega^{2} k^{2} L_{p}^{2}},
\end{aligned}
$$

where the equivalent input resistance $R_{T i}$ is

$$
R_{T i}=\frac{\omega^{2} L_{p}^{2} k^{2} R_{i}}{R_{i}^{2}+\omega^{2} k^{2} L_{p}^{2}},
$$

and the equivalent input reactance is

$$
X_{T i}=\frac{\omega^{3} L_{p}^{3} k^{2}(1-k)+\omega L_{p} R_{i}^{2}}{R_{i}^{2}+\omega^{2} k^{2} L_{p}^{2}} .
$$

From (12), the equivalent inductance is

$$
L_{T i}=\frac{X_{T i}}{\omega}=\frac{\omega^{2} L_{p}^{3} k^{2}(1-k)+L_{p} R_{i}^{2}}{R_{i}^{2}+\omega^{2} k^{2} L_{p}^{2}} .
$$

Fig. 1(c) shows the equivalent circuit of the Class-E ZVS inverter with a series resonant circuit composed of the resonant capacitor $C$, total resonant inductance $L=L_{e x t}+L_{T i}$, and loaded by the resistance $R_{T i}$. It must be noted that $L_{T i}$ and $R_{T i}$ are functions of the coupling coefficient $k$. For the Class$\mathrm{E}$ inverter to undergo ZVS, the load resistance must satisfy $0 \leq R_{T i} \leq R_{o p t}$, imposing a theoretical limit on the range 
of $k$ for maximum efficiency. The expressions for the external inductance $L_{\text {ext }}$ can be determined as follows. From Fig. 1(b),

$$
L_{1}=L_{e x t}+L_{l p}=L_{e x t}+(1-k) L_{p} .
$$

From the principles of impedance matching and transformation described in [7] for the $\pi 2 a$ circuit, we have

$$
X_{L 1}=\omega L_{1}=\left(Q_{L}-\sqrt{\frac{R_{i}}{R_{T i}}-1}\right) R_{T i},
$$

and

$$
X_{L 2}=\omega L_{2}=\omega L_{m}=\frac{R_{i}}{\sqrt{\frac{R_{i}}{R_{T i}}-1}} .
$$

The expression to determine the reactance of the shunt capacitor across the MOSFET is

$$
X_{C 1}=\frac{1}{\omega C_{1}}=\frac{\pi\left(\pi^{2}+4\right)}{8} R_{T i} .
$$

The value of the resonant capacitor can be determined using

$$
C=\frac{1}{\omega X_{C}}=\frac{1}{\omega\left[Q_{L}-\frac{\pi\left(\pi^{2}-4\right)}{16}\right] R_{T i}} .
$$

The expression to determine the choke inductance is

$$
L_{f}=2\left(\frac{\pi^{2}}{4}+1\right) \frac{R_{T i}}{4 f} .
$$

The above analysis holds true for the inverter loaded by a rectifier with reactive input impedance $Z_{i r}=R_{i r}+s L_{i r}$. A first-order approximation of input impedance of a singleended rectifier is equivalent to an input inductance $L_{i r}$ in series with the input resistance $R_{i r}$. Thus the total reactance, which includes the input inductance $L_{i r}$ and the secondary leakage inductance $L_{l s}$ can be canceled by tuning the value of the series capacitor $C_{s}$. A new expression for the series capacitance in the presence of the input inductance is

$$
C_{s}=\frac{1}{\omega_{s}^{2}\left(L_{l s}+L_{i r}\right)}=\frac{1}{4 \pi^{2} f_{s}^{2}\left[(1-k) L_{s}+L_{i r}\right]} .
$$

Simulation results demonstrating ZVS conditions with a series inductance are shown in the subsequent section.

\section{DESIGN EXAMPLE}

A Class-E ZVS inverter was designed for the following specifications: dc supply voltage $V_{I}=10 \mathrm{~V}$, output power of the inverter $P_{r i}=10 \mathrm{~W}$, and switching frequency $f_{s}=100 \mathrm{kHz}$. The quality factor is $Q_{L}=10$. The selfinductances of the primary and secondary coils are chosen as $L_{p}=L_{s}=24 \mu \mathrm{H}$ to give the turns ratio between the two coils $n=1$. From (1), the load resistance of the inverter at the secondary of the transformer shown in Fig. 1(a) is

$$
R_{i r}=\frac{8}{\pi^{2}+4} \frac{V_{I}^{2}}{P_{R i}} \approx 0.5768 \frac{10^{2}}{10}=5.76 \Omega .
$$

For $n=1$, using (6), we have

$$
R_{i}=n^{2} R_{i r}=5.76 \Omega .
$$

The coils are aligned to achieve a coupling coefficient of $k=$ 0.77 . Using (11), the equivalent input resistance is

$$
\begin{aligned}
R_{T i} & =\frac{\omega^{2} L_{p}^{2} k^{2} R_{i}}{R_{i}^{2}+\omega^{2} k^{2} L_{p}^{2}} \\
& =\frac{\left(2 \pi \times 10^{5}\right)^{2} \times\left(24 \times 10^{-6}\right)^{2} \times 0.77^{2} \times 5.76}{5.76^{2}+\left(2 \pi \times 10^{5}\right)^{2} \times 0.77^{2} \times\left(24 \times 10^{-6}\right)^{2}} \\
& =4.62 \Omega .
\end{aligned}
$$

The value of the equivalent input inductance using (13) is

$$
L_{T i}=\frac{\omega^{2} L_{p}^{3} k^{2}(1-k)+L_{p} R_{i}^{2}}{R_{i}^{2}+\omega^{2} k^{2} L_{p}^{2}}=9.2 \mu \mathrm{H} .
$$

Using (16), the value of the magnetizing inductance at $\omega=$ $\omega_{s}=2 \pi f_{s}$ is

$$
L_{m}=\frac{R_{i}}{\omega \sqrt{\frac{R_{i}}{R_{T i}}-1}}=\frac{5.76}{\omega \sqrt{\frac{5.76}{4.62}-1}}=18.48 \mu \mathrm{H} .
$$

The primary and secondary leakage inductances are equal as the turns ratio $n=1$ to give

$L_{l p}=L_{l s}=(1-k) L_{p}=(1-0.77) \times 24 \times 10^{-6}=5.52 \mu \mathrm{H}$.

The secondary-side resonant capacitance required to nullify the leakage inductance $L_{l s}$ as given in (5)

$$
\begin{aligned}
C_{s} & =\frac{n^{2}}{4 \pi^{2} f_{s}^{2}(1-k) L_{p}} \\
& =\frac{1}{4 \pi^{2} \times\left(10^{5}\right)^{2} \times(1-0.77) \times 24 \times 10^{-6}}=0.45 \mu \mathrm{F} .
\end{aligned}
$$

Using (15), the inductance $L_{1}$ is

$$
\begin{aligned}
L_{1} & =\frac{R_{T i}}{\omega}\left(Q_{L}-\sqrt{\frac{R_{i}}{R_{T i}}-1}\right) \\
& =\frac{4.62}{2 \pi \times 10^{5}}\left(10-\sqrt{\frac{5.76}{4.62}-1}\right)=69.97 \mu \mathrm{H} .
\end{aligned}
$$

Therefore, the external inductance is

$$
L_{e x t}=L_{1}-L_{l p}=69.97-5.52=64.45 \mu \mathrm{H} .
$$

The total inductance $L$ in the resonant circuit of the form shown in Fig. 1(c) is

$$
L=L_{\text {ext }}+L_{T i}=64.45+9.2=73.63 \mu \mathrm{H} .
$$

The resonant capacitor in series with $L$ is determined using (18) as

$$
\begin{aligned}
C & =\frac{1}{\omega\left[Q_{L}-\frac{\pi\left(\pi^{2}-4\right)}{16}\right] R_{T i}} \\
& =\frac{1}{2 \pi \times 10^{5} \times\left[10-\frac{\pi\left(\pi^{2}-4\right)}{16}\right] 4.62}=38.83 \mathrm{nF} .
\end{aligned}
$$


TABLE I

Values of Components Shown in Fig. 1(A) Used for Simulations

\begin{tabular}{|c|c|c|}
\hline Parameter & Calculated & Standard Value/ESR \\
\hline \hline DC supply voltage $V_{I}$ & $10 \mathrm{~V}$ & \\
Output power $P_{r i}$ & $10 \mathrm{~W}$ & \\
Switching frequency $f_{s}$ & $100 \mathrm{kHz}$ & \\
Coupling coefficient $k$ & 0.77 & \\
Choke inductor $L_{f}$ & $320.08 \mu \mathrm{H}$ & $360 \mu \mathrm{H} / 0.025 \Omega$ \\
Shunt capacitor $C_{1 e x t}$ & $63.043 \mathrm{nF}$ & $62 \mathrm{nF} / 0.01 \Omega$ \\
Resonant capacitor $C$ & $38.83 \mathrm{nF}$ & $39 \mathrm{nF} / 0.1 \Omega$ \\
Extra resonant inductor $L_{e x t}$ & $64.45 \mu \mathrm{H}$ & $65 \mu \mathrm{H} / 0.012 \Omega$ \\
Primary self-inductance $L_{p}$ & $24 \mu \mathrm{H}$ & \\
Secondary self-inductance $L_{s}$ & $24 \mu \mathrm{H}$ & \\
Secondary series capacitor $C_{s}$ & $0.45 \mu \mathrm{F}$ & $0.47 \mu \mathrm{F}$ \\
Load resistance $R_{i r}$ & $5.67 \Omega$ & $6 \Omega$ \\
\hline
\end{tabular}

Finally, the choke inductance is

$L_{f}=2\left(\frac{\pi^{2}}{4}+1\right) \frac{R_{T i}}{4 f}=2\left(\frac{\pi^{2}}{4}+1\right) \frac{4.62}{4 \times 10^{5}}=320.83 \mu \mathrm{F}$.

From [6], the voltage stress across the MOSFET is $V_{D S M} \approx$ $3.562 V_{I}=35.62 \mathrm{~V}$ and the current stress is $I_{D M} \approx$ $2.862 I_{I}=2.862 \times P_{I} / V_{I}=2.862 \times 1 \mathrm{~A}=2.862 \mathrm{~A}$. An IRF520 n-channel power MOSFET manufactured by International Rectifiers was selected. The properties of the MOSFET are: maximum drain current $I_{D M}=37 \mathrm{~A}$, maximum drain-to-source breakdown voltage $V_{B R R}=100 \mathrm{~V}$, drain-tosource resistance $r_{D S}=0.27 \Omega$, and output capacitance is $C_{o}=C_{\text {oss }}-C_{r s s}=0.150-0.034=0.117 \mathrm{nF}$. From (33), the value of the shunt capacitor is

$$
C_{1}=\frac{8}{\omega \pi\left(\pi^{2}+4\right) R_{T i}} \approx \frac{0.1836}{2 \pi \times 10^{5} \times 4.62}=63.16 \mathrm{nF} .
$$

The value of $C_{1}$ given in (33) is the total capacitance, which includes the output capacitance $C_{o}$ of the MOSFET. Therefore, the actual value of the shunt capacitance required is $C_{1 e x t}=$ $C_{1}-C_{o}=63.16-0.117=63.043 \mathrm{nF}$. The values of all the components, which have been calculated, their standard values used for simulations along with their equivalent series resistances are given in Table I.

\section{Simulation Results}

The circuit of the transformer-version of the Class-E zerovoltage switching (ZVS) inverter shown in Fig. 1(a) was simulated on SABER circuit simulator using the parameters and component values given in Table I. Simulations were performed first at the optimum coupling coefficient of $k=k_{\text {opt }}=0.77$ at which the inverter was designed. In addition, simulations were performed at coupling coefficients of $k=0.85>k_{\text {opt }}$ and $k=0.7<k_{\text {opt }}$ to realize the sensitivity of the inverter to variations in misalignment between the primary and secondary coils.

Fig. 2 shows the gate-to-source, drain-to-source, and drain current waveforms of the Class-E inverter at the optimum value of coupling coefficient $k_{o p t}=0.77$. Both zero-voltage and zero-derivative switching conditions were satisfied. Fig.

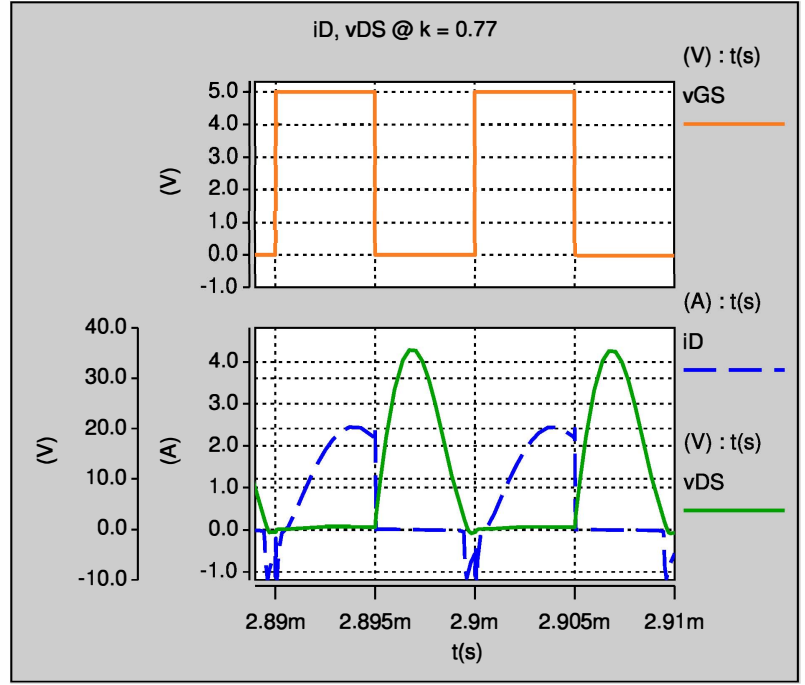

Fig. 2. Simulation results of switch current and voltage waveforms of the transformer-version of Class-E ZVS inverter at $k=0.77$ demonstrating both zero-voltage and zero-derivative switching.

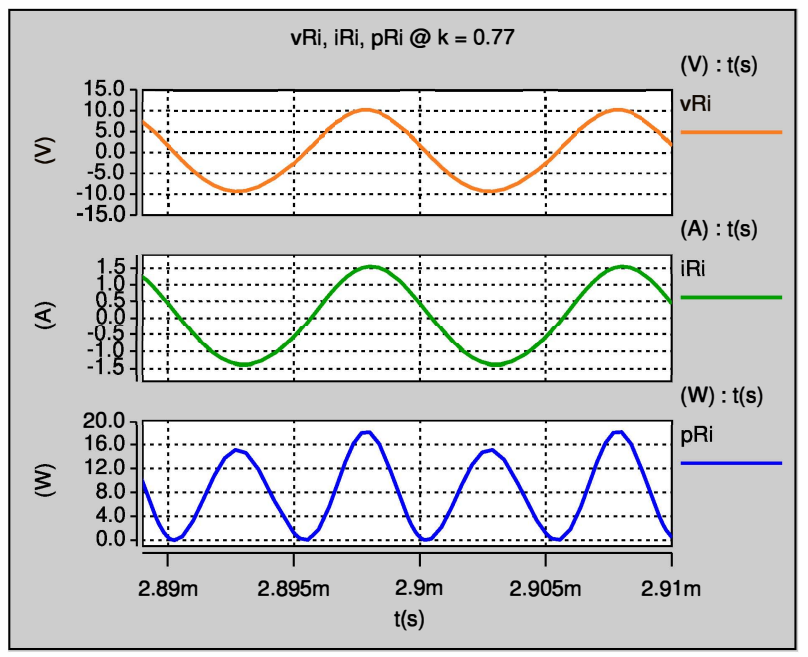

Fig. 3. Simulation results of output voltage, current, and power of the transformer-version of Class-E ZVS inverter at $k=0.77$.

3 shows the waveforms of the output current, output voltage, and output power. The amplitude of the output voltage was $V_{\text {Rim }}=10.25 \mathrm{~V}$ and the current was $I_{\text {Rim }}=1.52 \mathrm{~A}$. The average power delivered to the load resistance $R_{i r}$ was $P_{r i}=9.4 \mathrm{~W}$, while the design value is $10 \mathrm{~W}$. The average input power was determined as $P_{I}=9.6 \mathrm{~W}$. Therefore, the overall efficiency at $k_{o p t}=0.77$ was $\eta \approx 94.3 \%$.

Fig. 4 shows the switch voltages and current waveforms for the inverter at a coupling coefficient of $k=0.85$. One may observe that zero-voltage switching condition is not satisfied at this operating point. At $k=0.85>k_{o p t}$, the resistance $R_{T i}$ is lower than the optimum value of $4.62 \Omega$ for which the inverter was designed. Consequently, the ac current through the series resonant tank is high than that at $k=k_{o p t}$ and is negative at the turn-on instant. Fig. 5 shows the simulated waveforms 




Fig. 4. Simulation results of switch current and voltage waveforms of the transformer-version of Class-E ZVS inverter at $k=0.85$ demonstrating non zero-voltage switching operation.

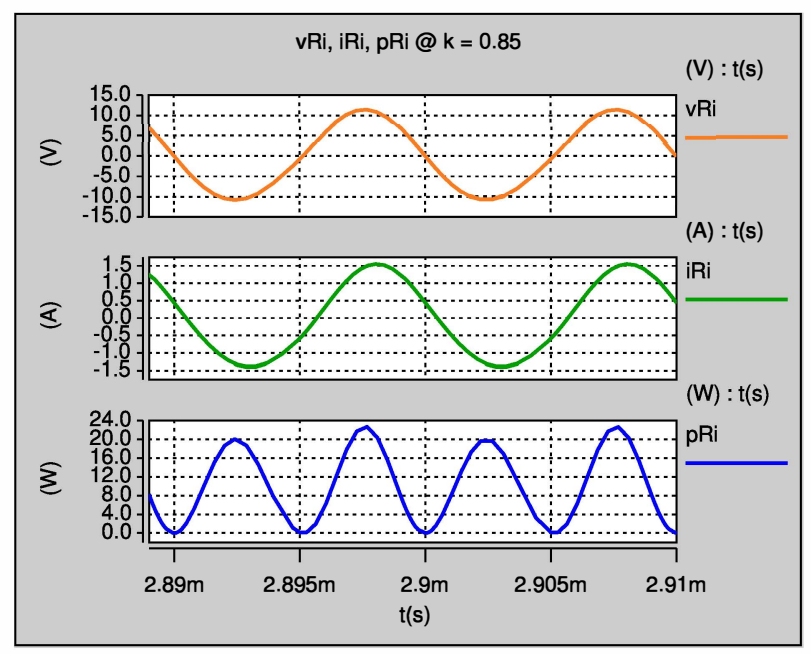

Fig. 5. Simulation results of output voltage, current, and power of the transformer-version of Class-E ZVS inverter at $k=0.85$.

of the output voltage, current, and power. The amplitude of the output voltage was $V_{\text {Rim }}=10.75 \mathrm{~V}$ and the current was $I_{\text {Rim }}=1.6 \mathrm{~A}$. The average output power was $P_{r i}=9.2 \mathrm{~W}$ and the average input power was $P_{I}=9.85 \mathrm{~W}$. The overall efficiency was measured as $\eta \approx 93.4 \%$. While the output power is higher at $k>k_{\text {opt }}$, the efficiency is lower due to off-nominal operation.

Fig. 6 shows the switch voltages and current waveforms for the inverter at a coupling coefficient of $k=0.7$. The drain-to-source voltage waveform undergoes both zero-voltage and zero-derivative switching. At $k<0.77$, the resistance $R_{T i}$ is lower than the optimum value $4.62 \Omega$, while the inductance $L_{T i}$ is high and limits the rate of rise of current. The output voltage, current, and power waveforms can be observed through Fig. 7. The amplitude of the output voltage

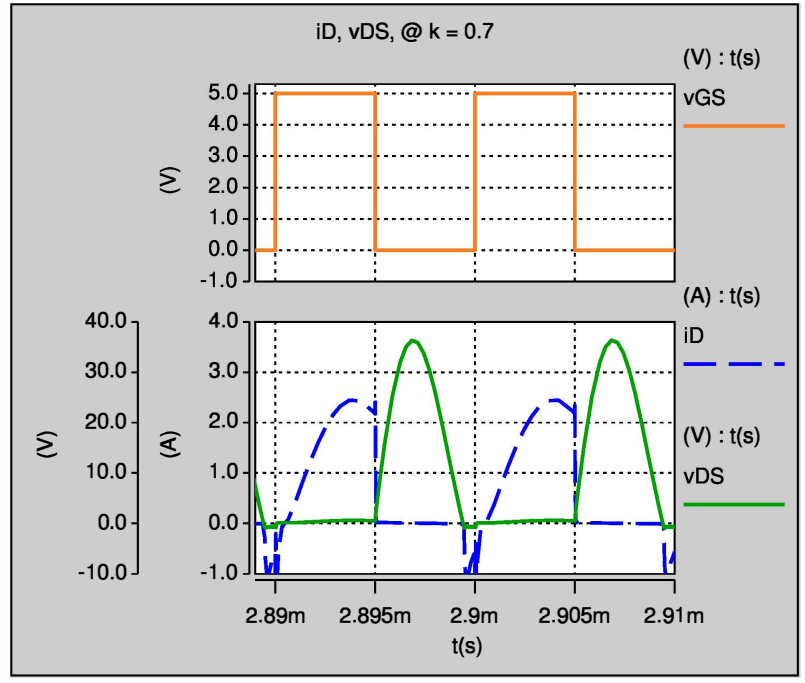

Fig. 6. Simulation results of switch current and voltage waveforms of the transformer-version of Class-E ZVS inverter at $k=0.7$ demonstrating both zero-voltage and zero-derivative switching.

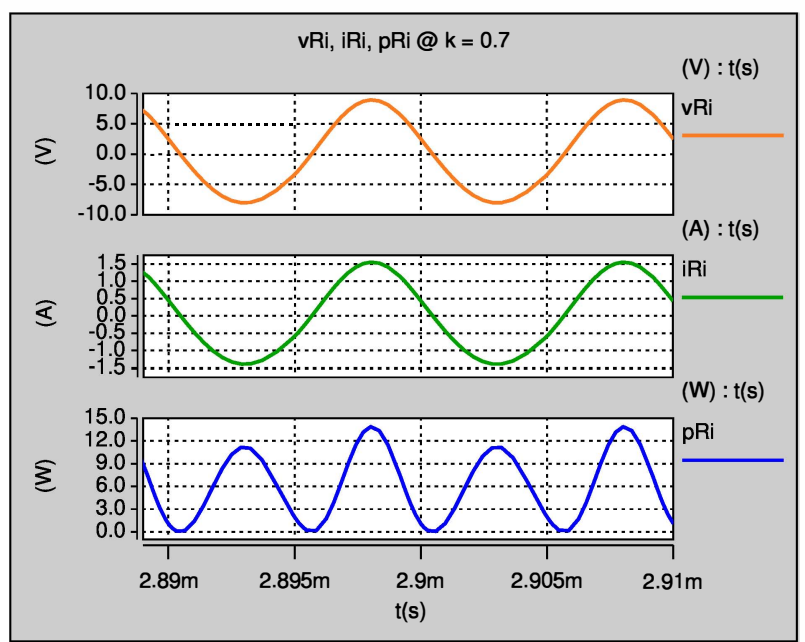

Fig. 7. Simulation results of output voltage, current, and power of the transformer-version of Class-E ZVS inverter at $k=0.7$.

has reduced to $V_{\text {Rim }}=8.5 \mathrm{~V}$ and the output current is $I_{\text {Rim }}=1.5 \mathrm{~A}$. The average output power was lower than that for $k=0.77$ and was measured as $P_{r i}=8.2 \mathrm{~W}$. The average input power was $P_{I}=8.92 \mathrm{~W}$. The overall efficiency was determined as $\eta \approx 92.12 \%$.

Finally, in the circuit shown in Fig. 1(a), the secondary of the transformer was modified by including the equivalent input inductance $L_{i r}$ of the rectifier in series with $R_{i r}$. The value of $L_{i r}$ was chosen as $30 \mu \mathrm{H}$. Using (20), the new value of series capacitance $C_{s}$ was determined as $71.3 \mathrm{nF}$. Fig. 8 shows the waveforms of the output voltage, output power, drain-to-source voltage, and drain current. The waveforms showed identical characteristics as those for the resistive load. Thus, the effect of any inductance on the secondary can be nullified by suitably adjusting the value of the series capacitance. 


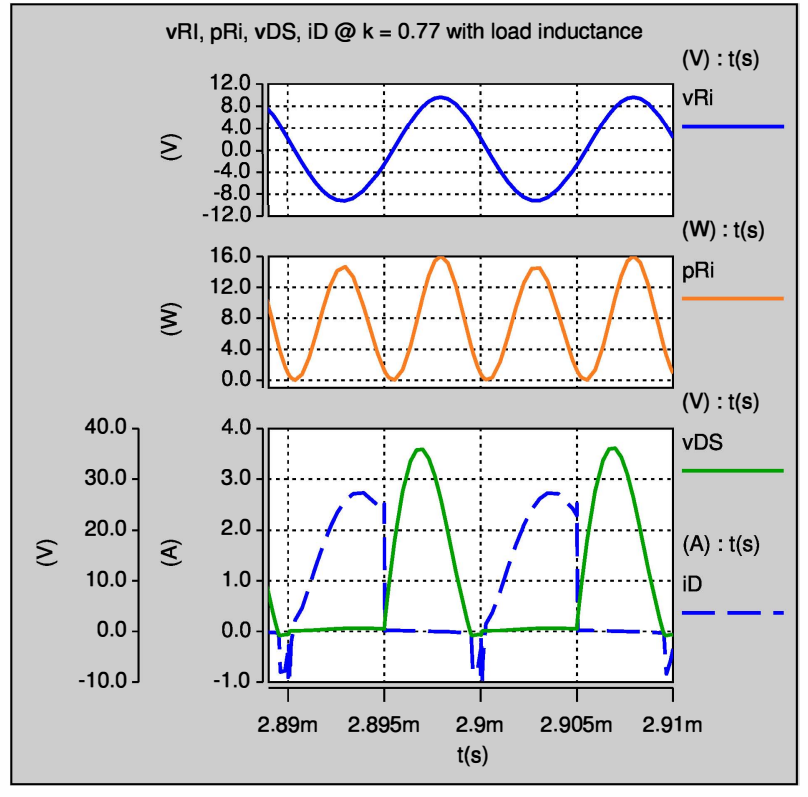

Fig. 8. Simulation results showing the output voltage, output power, switch current, and switch voltage of the transformer-version of Class-E ZVS inverter at $k=0.77$ including the input inductance of the rectifier.

\section{CONCLUSIONS}

This paper has introduced a procedure to design a ClassE ZVS inverter with a loosely-coupled transformer at a fixed coupling coefficient. The circuit is designed to satisfy both zero-voltage switching (ZVS) and zero-derivative switching (ZDS) conditions at optimum values of coupling coefficient and load resistance. It has been shown that, due to the magnetizing inductance of the transformer, the Class-E ZVS inverter behaves as an impedance inverter, which is known to increase the range of load resistance required for optimum operation. The circuit also resembles the Class-E inverter with a $\pi 2 a$ impedance matching topology. The magnetizing and the leakage inductances are absorbed into the main stage of the inverter. The design equations of the resonant components are provided in terms of coupling coefficient. This method can be adopted for the design of the Class-E inverter at any coupling coefficient. The Class-E ZVS inverter has been designed for a set of practical specifications. Using the theoretically predicted values of the components at an optimum coupling coefficient of $k=k_{\text {opt }}=0.77$, the circuit was built and tested on SABER circuit simulator. Simulation results have been presented to verify its performance at selected values of the coupling coefficients. The following results have been observed:

- The inverter achieves both ZVS and ZDS at the optimum coupling coefficient as shown in Fig. 2.

- At a coupling coefficient higher than the optimum value, the inverter does not achieve ZVS and is demonstrated in Fig. 4.

- Only ZVS is achieved for any coupling coefficient lower than its optimum value and is shown in Fig. 6.

\section{REFERENCES}

[1] M. K. Kazimierczuk and X. T. Bui, "Class E dc/dc converters with an inductive impedance inverter," IEEE Trans. Power Electron., vol. PE-4, no. 1, pp. 124-135, January 1989.

[2] M. K. Kazimierczuk and X. T. Bui, "Class E dc/dc converters with a capacitive impedance inverter," IEEE Trans. Ind. Electron., vol. IE-36, no. 8, pp. 425-433, August 1989.

[3] M. K. Kazimierczuk and J. Jozwik, "DC/DC converter with class E zerovoltage-switching inverter and class E zero-current-switching rectifier," IEEE Trans. Circ. and Syst., vol. 36, no. 11, pp. 1485-1488, Nov 1989.

[4] M. K. Kazimierczuk, N. Thirunarayan, and S. Wang, "Analysis of series parallel resonant converter," IEEE Trans. Aerosp. and Electron. Syst., vol. 29 , no. 1 , pp. 88-99, 1993.

[5] J. Modzelewski, "Optimum and sub-optimum operation of highfrequency Class-D zero-voltage-switching tuned power amplifier," Bull. Polish Acad. Sci., Tech. Sci., vol. 46, no. 4, pp. 458-473, Apr. 1998.

[6] M. K. Kazimierczuk, RF Power Amplifiers, 2nd Ed., John Wiley Sons, Chichester, UK, 2014.

[7] M. K. Kazimierczuk and D. Czarkowski, Resonant Power Converters, 2nd Ed., John Wiley Sons, Hoboken, NJ, 2012.

[8] H. Sarnago, O. Lucia, A. Mediano, and J. M. Burdio, "Class-D/DE dual mode-operation resonant converter for improved-efficiency domestic induction heating system," IEEE Trans. Power Electron., vol. 28, no. 3, pp. 1274-1285, Mar. 2013.

[9] D. Murthy-Bellur, A. Bauer, W. Kerin, and M. K. Kazimierczuk, "Inverter using loosely coupled inductors for wireless power transfer," Proc. IEEE Intl. Midwest Symp. Circ. Syst., Boise, ID, USA, Aug. 2012, pp.1164-1167.

[10] A. Ayachit and M. K. Kazimierczuk, "Transfer functions of a transformer at different coupling coefficients," IET Circ. Devices Syst., April 2016, doi: 10.1049/iet-cds.2015.0147.

[11] A. Ayachit, D. K. Saini, T. Suetsugu, and M. K. Kazimierczuk, "Threecoil wireless power transfer system using Class-E2 resonant dc-dc converter," Proc. IEEE Intl. Telecommunications Energy Conf., INTELEC, Yokohama, Japan, October 2015, pp. 1116-1119.

[12] Z. H. Ye, Y. Sun, X. Dai, C. S. Tang, Z. H. Wang, and Y. G. Su, "Energy efficiency analysis of U-Coil wireless power transfer system," IEEE Trans. Power Electron., vol. 31, no. 7, pp. 4809-4817, July 2016.

[13] K. Inoue, T. Nagashima, X. Wei, and H. Sekiya, "Design of highefficiency inductive-coupled wireless power transfer system with classDE transmitter and class-E rectifier,' Proc. IEEE Industrial Electronics Society, Vienna, Austria, Nov. 2013, pp. 613-618.

[14] M. Hayati, A. Lotfi, M. K. Kazimierczuk, and H. Sekiya, "Analysis and design of class-E power amplifier with MOSFET parasitic linear and nonlinear capacitances at any duty ratio," IEEE Trans. Power Electron., vol. 28, no. 11, pp. 5222-5232, Nov. 2013.

[15] A. Mediano and P. Molina, "Frequency limitation of a high-efficiency class E tuned RF power amplifier due to a shunt capacitance," Proc. IEEE MTT-S Intl. Microwave. Symp. Dig., Anaheim, CA, USA, Jun. 1999, pp. 13-19.

[16] A. Mediano, P. Molina, and J. Navarro, "Class E RF/microwave power amplifier: Linear Equivalent of transistors nonlinear output capacitance, normalized design and maximum operating frequency versus output capacitance," Proc. IEEE MTT-S Int. Microw. Symp. Dig., Boston, MA, USA, Jun., 2000, pp. 783-786.

[17] X. Wei, H. Sekiya, S. Kuroiwa, T. Suetsugu, and M. K. Kazimierczuk, "Design of class-E amplifier with MOSFET linear gate-to-drain and nonlinear drain-to-source capacitances," IEEE Trans. Circuits Syst.-I, vol. 58, no. 10 , pp. 2556-2565, Oct. 2011

[18] T. Suetsugu and M. K. Kazimierczuk, "Maximum operating frequency of class-E amplifier at any duty ratio," IEEE Trans. Circ. Syst.-II, vol. 55, no. 8, pp. 768-770, Aug. 2008.

[19] S. C. Wong and C. K. Tse, "Design of symmetrical Class E power amplifiers for very low harmonic-content applications," IEEE Trans. Circ. Syst.-I, vol. 52, no. 8, pp. 1684-1690, Aug. 2005.

[20] M. Kazimierczuk and K. Puczko, "Exact analysis of class E tuned power amplifier at any Q and switch duty cycle," IEEE Trans. Circ. Syst., vol. 34, no. 2, pp. 149-159, Feb. 1987. 